\title{
Viscoelastic and Orientational Relaxation of Linear and Ring Rouse Chains undergoing Reversible End-Association and Dissociation.
}

\author{
Youngdon Kwon \\ School of Chemical Engineering, Sungkyunkwan University \\ 300 Cheoncheon-dong, Jangan-gu, Suwon, Gyeonggi-do 440-746, Korea \\ Yumi Matsumiya and Hiroshi Watanabe* \\ Institute for Chemical Research, Kyoto University, Uji, Kyoto 611-0011, Japan
}

In the followings, equations with and without an alphabetical heading in their number, such as eq A1 and eq 47, refer to those in the Appendices and main text, respectively. The same applies to sections (for example, section 2-3 refers to the section in the main text).

\section{Appendix A. Direct Calculation of Orientational Correlation Function.}

A1. In the absence of the reaction. In the absence of association/dissociation reaction, we can calculate the orientational correlation function of the linear and ring chains very simply (simpler than the calculation explained in section 2-3). In this simple calculation, we consider the boundary condition (eqs 13 and 21 in the main text) to expand this function as

$$
\text { Linear: } \begin{aligned}
S^{[\mathrm{L}]}(n, m, t) & =\sum_{p=1}^{N} \sum_{q=1}^{N} X_{p q}(t) \sin \left(\frac{p \pi n}{N}\right) \sin \left(\frac{q \pi m}{N}\right) \\
\text { Ring: } S^{[\mathrm{R}]}(n, m, t) & =\sum_{p=1}^{N / 2} \sum_{q=1}^{N / 2} Y_{p q}(t) \sin \left(\frac{2 p \pi n}{N}\right) \sin \left(\frac{2 q \pi m}{N}\right) \\
& +\sum_{p=1}^{N / 2} \sum_{q=1}^{N / 2} Z_{p q}(t) \cos \left(\frac{2 p \pi n}{N}\right) \cos \left(\frac{2 q \pi m}{N}\right)
\end{aligned}
$$

The expansion coefficients are equivalent to the averaged shear components of the dyadic of the eigenmode amplitudes defined in section 2-3: $X_{p q}(t)=b^{-2}<\boldsymbol{\Xi}_{p}(t) \boldsymbol{\Xi}_{q}(t)>_{x y}, Y_{p q}(t)=b^{-2}<\boldsymbol{\Psi}_{p}(t) \boldsymbol{\Psi}_{q}(t)>_{x y}$, and $Z_{p q}(t)=b^{-2}<\mathbf{A}_{p}^{[\mathrm{R}]}(t) \mathbf{A}_{q}^{[\mathrm{R}]}(t)>_{x y}$. Because the Brownian force having the isotropic white-noise character does not affect these shear components, we can combine eqs A1 and A2 with the Rouse equation of motion, eq 10 in the main text, to formulate the time evolution of $X_{p q}(t), Y_{p q}(t)$, and $Z_{p q}(t)$ in a differential form, ${ }^{1,2}$ 
Linear: $\dot{X}_{p q}(t)=-\frac{p^{2}+q^{2}}{\tau_{1}} X_{p q}(t)$

Ring: $\dot{Y}_{p q}(t)=-\frac{4\left(p^{2}+q^{2}\right)}{\tau_{1}} Y_{p q}(t), \quad \dot{Z}_{p q}(t)=-\frac{4\left(p^{2}+q^{2}\right)}{\tau_{1}} Z_{p q}(t)$

( $\tau_{1}$ is the slowest Rouse relaxation time given by eq 16.) Considering the initial conditions, eqs 18 and 24, eqs A3 and A4 are straightforwardly solved to give the well-known Rouse results, eqs 19 and 25 in the main text.

A2. In the presence of the reaction. The above method of calculating the expansion coefficients of $S^{[c]}(n, m, t)$ is useful even in the presence of the association/dissociation reaction, given that the reaction rate is not explicitly correlated with those coefficients describing the chain conformation. ${ }^{3}$ In the absence of this explicit correlation, the time evolution of $Z_{\alpha \beta}(t)$ of the ring chain (coefficient of the cosine term in eq A2), for example, can be generally expressed as

$$
\dot{Z}_{\alpha \beta}(t)=-\frac{4\left(\alpha^{2}+\beta^{2}\right)}{\tau_{1}} Z_{\alpha \beta}(t)-\frac{1}{\tau_{\mathrm{ds}}} Z_{\alpha \beta}(t)+\frac{1}{\tau_{\mathrm{ds}}} \sum_{p, q \geq 1} \eta_{p \alpha} \eta_{q \beta} X_{p q}(t)
$$

Here, the second term in the right-hand-side denotes disappearance of the ring chain (conversion into the linear chain), and the third term, creation of a ring chain through association of the linear chain ends. This creation is equivalent to mapping of the linear chain conformation specified by eq A1 onto the ring chain, so that the creation term includes the Fourier expansion coefficient $\eta_{p \alpha}$ of the sine eigenfunction for the linear chain, $\sin (p \pi n / N)$, with respect to the cosine eigenfunction for the ring chain, $\cos (2 \alpha \pi n / N)$,

$$
\eta_{p \alpha}=\frac{4}{\pi} \frac{p}{p^{2}-4 \alpha^{2}}(p=\text { odd }), \quad \eta_{p \alpha}=0(p=\text { even })
$$

(This $\eta_{p \alpha}$ is identical to that specified by eq 31 for $p=$ odd integer.) Expressions similar to eq A5 are obtained also for $X_{\alpha \beta}$ and $Y_{\alpha \beta}$.

The above method may require a large effort in the calculation of the summation (cf. eq A5) but is conceptually straightforward. In fact, the method was successfully utilized to analyze the relaxation of mono-functionally associating linear chains and their dimers. ${ }^{3}$

However, unfortunately, that method is not easily applicable to the system examined in this study. Namely, the end-association of the telechelic linear chain occurs only when two ends of this chain come into close proximity, quite possibly within a distance $<b$ (subchain step length). In the strict case, the ring formation requires the bond vector of the linear chain $\mathbf{u}^{[\mathrm{L}]}(n, t)$ to satisfy a relationship equivalent to the boundary condition for the ring chain (cf. eq 21b), 


$$
\int_{0}^{\mathrm{N}} \mathrm{d} n \mathbf{u}^{[\mathrm{L}]}(n, t)=\mathbf{0}
$$

Consequently, the ring is formed only when the eigenmode amplitudes $\boldsymbol{\Xi}_{p}(t)$ of the linear chain, defined by eq 14 in the main text, satisfy a condition (identical to eq 46),

$$
\sum_{p=\text { odd }}^{N} \frac{\boldsymbol{\Xi}_{p}(t)}{p}=\mathbf{0}
$$

This condition, expressed for $\boldsymbol{\Xi}_{p}(t)$ without any averaging, cannot be easily incorporated in the time evolution equation of the expansion coefficients $X_{p q}, Y_{p q}$, and $Z_{p q}$ that are defined as the ensemble-averaged shear components of dyadic of the eigenmode amplitudes. (This incorporation should require us to introduce a complicated Lagrange factor (Lagrange multiplier) in those time evolution equations.) Thus, in this study, we have analyzed the time evolution of the unaveraged mode amplitudes, not the averaged dyadic, to calculate the orientational correlation function and viscoelastic relaxation function of the linear and ring chains at the association/dissociation equilibrium. 


\section{Appendix B. Mode Expansion of Subchain Bond Vector}

B1. Expansion in the absence of association/dissociation reaction. In the absence of the reaction, the subchain bond vectors $\mathbf{u}(n, t)$ of the linear and ring chains are expanded into respective Rouse eigenmodes, as shown in eqs 14 and 22 in the main text. The distribution function of the mode amplitudes at equilibrium, $W_{\mathrm{eq}}$, is specified in terms of $\mathbf{u}_{\mathrm{eq}}(n)$ at equilibrium as ${ }^{4}$

$$
W_{\text {eq }}=C \exp \left(-\frac{E}{k_{B} T}\right)=C \exp \left(-\frac{\kappa}{2 k_{B} T}\left\{\int_{0}^{N} \mathbf{u}_{\text {eq }}^{2}(n) \mathrm{d} n\right\}\right)
$$

where $E=(\kappa / 2) \int_{0}^{N} \mathbf{u}_{\mathrm{eq}}^{2}(n) \mathrm{d} n$ indicates the elastic energy of the chain having the subchain spring constant $\kappa$ (cf. eq 11 in the main text), and $C$ is a normalization constant. From eq B1 combined with eqs 11,14 and $22, W_{\text {eq }}$ is explicitly expressed in a Gaussian form for the mode amplitudes at equilibrium, $\boldsymbol{\Xi}_{p}^{\mathrm{eq}}$ for the linear chain and $\boldsymbol{\Psi}_{\alpha}^{\mathrm{eq}}$ and $\mathbf{A}_{\alpha}^{\mathrm{eq}}$ for the ring chain:

For linear chain: $W_{e q}\left(\left\{\boldsymbol{\Xi}^{\mathrm{eq}}\right\}\right)=C \exp \left(-\frac{3 N}{4 b^{2}} \sum_{p=1}^{N}\left(\boldsymbol{\Xi}_{p}^{\mathrm{eq}}\right)^{2}\right)$

For ring chain: $W_{e q}\left(\left\{\Psi^{\mathrm{eq}}\right\},\left\{\mathbf{A}^{\mathrm{eq}}\right\}\right)=C \exp \left(-\frac{3 N}{4 b^{2}} \sum_{\alpha=1}^{N / 2}\left\{\left(\Psi_{\alpha}^{\mathrm{eq}}\right)^{2}+\left(\mathbf{A}_{\alpha}^{\mathrm{eq}}\right)^{2}\right\}\right)$

On application of the step shear strain $\gamma$ at $t=0, \mathbf{u}_{\mathrm{eq}}(n)$ is assumed to be affinely deformed to $\mathbf{u}(n, 0)$ $=\mathbf{D} \cdot \mathbf{u}_{\mathrm{eq}}(n)$, where $\mathbf{D}$ is the shear deformation tensor given by ${ }^{4,5}$

$$
\mathbf{D}=\left[\begin{array}{lll}
1 & \gamma & 0 \\
0 & 1 & 0 \\
0 & 0 & 1
\end{array}\right]
$$

Consequently, the mode amplitudes at $t=0$ are specified as $\boldsymbol{\Xi}_{p}(0)=\mathbf{D} \cdot \boldsymbol{\Xi}_{p}^{\mathrm{eq}}, \Psi_{\alpha}(0)=\mathbf{D} \cdot \Psi_{\alpha}^{\mathrm{eq}}$, and $\mathbf{A}_{\alpha}(0)=\mathbf{D} \cdot \mathbf{A}_{\alpha}^{\text {eq }}$. Thus, for the averaged dyadic, $\left\langle\boldsymbol{\Xi}_{p} \boldsymbol{\Xi}_{q}\right\rangle$, the initial value of the shear component is obtained from eq B2 as

$$
\left\langle\boldsymbol{\Xi}_{p}(0) \boldsymbol{\Xi}_{q}(0)\right\rangle_{x y}=\int_{-\infty}^{\infty}\left(\mathbf{D} \cdot \boldsymbol{\Xi}_{p}^{\mathrm{eq}}\right)_{x}\left(\mathbf{D} \cdot \boldsymbol{\Xi}_{q}^{\mathrm{eq}}\right)_{y} W_{e q}\left(\left\{\boldsymbol{\Xi}^{\mathrm{eq}}\right\}\right) \prod_{\alpha} \mathrm{d} \boldsymbol{\Xi}_{\alpha}^{\mathrm{eq}}=\frac{2 \gamma b^{2}}{3 N} \delta_{p q}
$$

Similarly, from eq B3, we find

$$
\left\langle\Psi_{\alpha}(0) \Psi_{\beta}(0)\right\rangle_{x y}=\left\langle\mathbf{A}_{\alpha}(0) \mathbf{A}_{\beta}(0)\right\rangle_{x y}=\frac{2 \gamma b^{2}}{3 N} \delta_{\alpha \beta}
$$

In addition, from eqs $\mathrm{B} 2$ and $\mathrm{B} 3$, we also note

$$
\left\langle\mathbf{A}_{\alpha}(0) \boldsymbol{\Psi}_{\beta}(0)\right\rangle_{x y}=\left\langle\boldsymbol{\Xi}_{p}(0) \mathbf{A}_{\alpha}(0)\right\rangle_{x y}=\left\langle\boldsymbol{\Xi}_{p}(0) \boldsymbol{\Psi}_{\alpha}(0)\right\rangle_{x y}=0 \text { for any } \alpha, \beta, p
$$


These simple expressions of the initial values result from the Gaussian form of the distribution functions specified by eqs B2 and B3.

B2. Expansion in the presence of association/dissociation reaction. The subchain bond vector of the ring chain can be expanded with respect to its Rouse eigenfunctions (in the form of eq 22 in the main text) irrespective of the reaction. Thus, the initial condition of the mode amplitude for the ring chain is specified by eqs B6 and B7 even in the presence of the reaction.

The situation is different for the linear chain. Because the end-association of the linear chain occurs only when its Rouse expansion coefficients $\boldsymbol{\Xi}_{p}(t)$ satisfy eq 46 , the analysis of the linear chain dynamics cannot be simply achieved if the subchain bond vector is expanded with respect to the sinusoidal Rouse eigenfunctions $\sin (p \pi n / N)$, as explained in Appendix A2. Thus, in the analysis, the contribution of the odd sine modes to the bond vector, $\mathbf{u}_{\text {odd }}^{[\mathrm{L}]}(n, t)=\sum_{p=\text { odd }}^{N} \boldsymbol{\Xi}_{p}(t) \sin (p \pi n / N)$, needs to be re-expanded with respect to cosine functions as $\mathbf{u}_{\text {odd }}^{[\mathrm{L}]}(n, t)=\sum_{\alpha=0}^{\infty} \mathbf{B}_{\alpha}(t) \cos (2 \alpha \pi n / N)$; see eq 47 in the main text. Comparing these two expressions of $\mathbf{u}_{\text {odd }}^{[\mathrm{L}]}(n, t)$ and making Fourier cosine integral of the former expression, we find

and

$$
\alpha=0: \quad \mathbf{B}_{0}(t)=\frac{1}{N} \int_{0}^{N} \mathbf{u}_{\text {odd }}^{[\mathrm{L}]}(n, t) \mathrm{d} n=\sum_{p=\text { odd }}^{N} \frac{2}{p \pi} \mathbf{\Xi}_{p}(t)
$$

$$
\alpha \geq 1: \quad \mathbf{B}_{\alpha}(t)=\frac{2}{N} \int_{0}^{N} \mathbf{u}_{\text {odd }}^{[\mathrm{L}]}(n, t) \cos \left(\frac{2 \alpha \pi n}{N}\right) \mathrm{d} n=\sum_{p=o d d}^{N} \eta_{p \alpha} \boldsymbol{\Xi}_{p}(t)
$$

with the Fourier expansion coefficient $\eta_{p \alpha}$ being given by

$$
\eta_{p \alpha}=\frac{4}{\pi} \frac{p}{p^{2}-4 \alpha^{2}}(p=\text { odd }, \alpha \geq 1)
$$

Similarly, we obtain

$$
p=\text { odd: } \Xi_{p}(t)=\frac{2}{N} \int_{0}^{N} \mathbf{u}_{\text {odd }}^{[\mathrm{L}]}(n, t) \sin \left(\frac{p \pi n}{N}\right) \mathrm{d} n=\frac{4}{p \pi} \mathbf{B}_{0}(t)+\sum_{\alpha=1}^{\infty} \eta_{p \alpha} \mathbf{B}_{\alpha}(t)
$$

The cosine expansion series, $\mathbf{u}_{\text {odd }}^{[\mathrm{L}]}(n, t)=\sum_{\alpha=0}^{\infty} \mathbf{B}_{\alpha}(t) \cos (2 \alpha \pi n / N)$, has a value $\sum_{\alpha=0}^{\infty} \mathbf{B}_{\alpha}(t)$ at the linear chain ends, $n=0, N$. From eqs $\mathrm{B} 8$ and $\mathrm{B} 9$, this value is expressed as

$$
\sum_{\alpha=0}^{\infty} \mathbf{B}_{\alpha}(t)=\sum_{p=\text { odd }}^{N}\left\{\frac{2}{p \pi}+\sum_{\alpha=1}^{\infty} \eta_{p \alpha}\right\} \boldsymbol{\Xi}_{p}(t)=\sum_{p=\text { odd }}^{N}\left\{\frac{2}{p \pi}-\frac{p}{\pi} \sum_{\alpha=1}^{\infty} \frac{1}{\alpha^{2}-(p / 2)^{2}}\right\} \boldsymbol{\Xi}_{p}(t)
$$

For $p=$ odd integer, the summation $\sum_{\alpha=1}^{\infty}\left\{\alpha^{2}-(p / 2)^{2}\right\}^{-1}$ appearing in eq B11 is equal to $2 / p^{2}$, as noted from a mathematical formula, ${ }^{6}$ 


$$
\sum_{\alpha=1}^{\infty} \frac{1}{\alpha^{2}-x^{2}}=\frac{1}{2 x^{2}}-\frac{\pi}{2 x} \cot (\pi x)
$$

(Note that $\cot (\pi x)=0$ for $x=p / 2$ with $p=$ odd integer.) Thus, eq B11 indicates $\sum_{\alpha=0}^{\infty} \mathbf{B}_{\alpha}(t)=\mathbf{0}$ irrespective of the $t$ dependence of $\boldsymbol{\Xi}_{p}(t)$. Namely, the expansion series $\sum_{\alpha=0}^{\infty} \mathbf{B}_{\alpha}(t) \cos (2 \alpha \pi n / N)$ introduced for the linear chain is always equal to $\mathbf{0}$ for $n=0$ and $N$, thereby satisfying the boundary condition for the linear chain, eq 13 in the main text.

B3. Initial value of average dyadic $<\mathbf{B B}>$. If the amplitudes of the cosine modes introduced for the linear chain, $\mathbf{B}_{\alpha}(t)$ with $\alpha \geq 0$, is subjected to no constraint, their equilibrium distribution function, specified by eq B1, has the Gaussian form similar to eqs B2 and B3 thereby giving simple expressions of the initial values of the dyadic averages, $\left\langle\mathbf{B}_{0}(0) \mathbf{B}_{\alpha}(0)\right\rangle_{x y}=\left(b^{2} \gamma / 3 N\right) \delta_{0 \alpha}$ and $\left\langle\mathbf{B}_{\alpha}(0) \mathbf{B}_{\beta}(0)\right\rangle_{x y}=\left(2 b^{2} \gamma / 3 N\right) \delta_{\alpha \beta}(\alpha, \beta \geq 1)$.

However, the amplitudes are actually subjected to the constraint reflecting the boundary condition of the linear chain, $\sum_{\alpha=0}^{\infty} \mathbf{B}_{\alpha}(t)=\mathbf{0}$ (that is automatically guaranteed when the sine and cosine expansion series for $\mathbf{u}_{\text {odd }}^{[\mathrm{L}]}(n, t), \sum_{p=\text { odd }}^{N} \boldsymbol{\Xi}_{p}(t) \sin (p \pi n / N)$ and $\sum_{\alpha=0}^{\infty} \mathbf{B}_{\alpha}(t) \cos (2 \alpha \pi n / N)$, are equated to each other; see Appendix B2). For this case, $\mathbf{B}_{\alpha}(0)$ and $\mathbf{B}_{\beta}(0)$ with all $\alpha$ and $\beta$ values have complicated correlation and the above simple expressions of $\left\langle\mathbf{B}_{\alpha}(0) \mathbf{B}_{\beta}(0)\right\rangle_{x y}$ lose their validity. This correlation is described by a non-Gaussian distribution function, $W_{e q}\left(\left\{\mathbf{B}_{\alpha}^{\mathrm{eq}} ; \alpha \geq 1\right\}\right)=$ $C \exp \left\{-\left(3 N / 4 b^{2}\right)\left[2 \Sigma_{\alpha, \beta \geq 1} \mathbf{B}_{\alpha}^{\mathrm{eq}} \bullet \mathbf{B}_{\beta}^{\mathrm{eq}}+\Sigma_{\alpha \geq 1}\left(\mathbf{B}_{\alpha}^{e q}\right)^{2}\right]\right\}$, calculated from eq B1 combined with the cosine expansion form with the constraint, $\mathbf{u}_{\text {odd }}^{[\mathrm{L}]}(n, t)=\sum_{\alpha=1}^{\infty} \mathbf{B}_{\alpha}(t)\{\cos (2 \alpha \pi n / N)-1\}$ (eq 49 in the main text). Expression of $\left\langle\mathbf{B}_{\alpha}(0) \mathbf{B}_{\beta}(0)\right\rangle_{x y}$ can be obtained from this $W_{e q}\left(\left\{\mathbf{B}_{\alpha}^{\mathrm{eq}} ; \alpha \geq 1\right\}\right)$ only after tedious calculation. In section 2-4-4 in the main text, we avoid this difficulty by utilizing eq B9a (at $t$ $=0$ ) to express $\mathbf{B}_{\alpha}(0)$ in terms of the well-behaving $\boldsymbol{\Xi}_{p}(0)$ (characterized by eq B5) to formulate $\mathbf{u}_{\text {odd }}^{[\mathrm{L}]}(n, t) \quad\left(=\sum_{\alpha=1}^{\infty} \mathbf{B}_{\alpha}(t)\{\cos (2 \alpha \pi n / N)-1\}\right)$ and $\quad \mathbf{u}_{\cos }^{[\mathrm{R}]}(n, t) \quad\left(=\sum_{\alpha=1}^{N / 2} \mathbf{A}_{\alpha}(t) \cos (2 \alpha \pi n / N)\right)$. 


\section{Appendix C. Rouse Evolution of Coefficients $B_{\alpha}$ Appearing in Eq 47 in the Main Text.}

For the cosine expansion coefficients $\mathbf{B}_{\alpha}$ for the linear chain appearing in eq 47 in the main text, we here consider the time evolution due only to the Rouse dynamics.

For $\mathbf{B}_{0}(t)(\alpha=0)$, we can specify the first order time derivative with the aid of eq B8 (in Appendix B2) and the Rouse evolution equation for $\boldsymbol{\Xi}_{p}$ (eq 15 in the main text):

$$
\dot{\mathbf{B}}_{0}(t)=\sum_{p=\text { odd }}^{N} \frac{2}{p \pi} \dot{\boldsymbol{\Xi}}_{p}(t)=-\frac{2}{\pi \tau_{1}} \sum_{p=\text { odd }}^{N} p \boldsymbol{\Xi}_{p}(t)
$$

Here, we have omitted a term linear to the Brownian force $\mathbf{F}_{\mathrm{B}}$ because of its isotropic white-noise character, as explained for eqs 27 and 28 in the main text. The coefficient $\boldsymbol{\Xi}_{p}(t)$ appearing in eq $\mathrm{C} 1$ can be expressed in terms of $\mathbf{B}_{\beta}(t)(\beta \geq 0)$, as shown by eq B10. Then, eq $\mathrm{C} 1$ becomes

$$
\dot{\mathbf{B}}_{0}(t)=-\frac{8}{\pi^{2} \tau_{1}} \sum_{\beta=0}^{\infty}\left\{\sum_{p=\text { odd }}^{N} \frac{p^{2}}{p^{2}-4 \beta^{2}}\right\} \mathbf{B}_{\beta}(t)
$$

The summation, $\sum_{p=\text { odd }}^{N} p^{2} /\left(p^{2}-4 \beta^{2}\right)$, is taken for $1 \leq p$ (odd) $\leq N$, but we can satisfactorily approximate this summation for $N \gg>1$ (considered in this study) as

$$
\sum_{p=\text { odd }}^{N} \frac{p^{2}}{p^{2}-4 \beta^{2}}=\sum_{p=\text { odd }}^{N}\left(1+\frac{4 \beta^{2}}{p^{2}-4 \beta^{2}}\right)=\frac{N}{2}
$$

Here, we have utilized a relationship $\sum_{p=\text { odd }}^{N} 1=N / 2$ (for $N>>1$ ) and also a mathematical formula, ${ }^{6}$

$$
\sum_{p=\text { odd }}^{\infty} \frac{x^{2}}{p^{2}-x^{2}}=\frac{\pi x}{4} \tan \left(\frac{\pi x}{2}\right)
$$

(This summation is equal to zero for $x=$ even integer including $x=0$ ). From eq C3, eq C2 is rewritten as

$$
\dot{\mathbf{B}}_{0}(t)=-\frac{4 N}{\pi^{2} \tau_{1}} \sum_{\beta=0}^{\infty} \mathbf{B}_{\beta}(t)
$$

This equation is shown as eq 50a (combined with eq 51) in the main text.

For $\mathbf{B}_{\alpha}(t)$ with $\alpha \geq 1$, the first order derivative is similarly obtained from eq B9 (Appendix B2) and eq 15 (main text):

$$
\dot{\mathbf{B}}_{\alpha}(t)=\sum_{p=\mathrm{odd}}^{N} \eta_{p \alpha} \dot{\boldsymbol{\Xi}}_{p}(t)=-\frac{4}{\pi \tau_{1}} \sum_{p=\mathrm{odd}}^{N} \frac{p^{3}}{p^{2}-4 \alpha^{2}} \mathbf{\Xi}_{p}(t)
$$


With the aid of the expression of $\boldsymbol{\Xi}_{p}(t)$ in terms of $\mathbf{B}_{\beta}(t)$ (eq B10), eq C6 becomes

$$
\dot{\mathbf{B}}_{\alpha}(t)=-\frac{16}{\pi^{2} \tau_{1}} \sum_{\beta=0}^{\infty} I_{\alpha \beta} \mathbf{B}_{\beta}(t)
$$

with

$$
\begin{aligned}
I_{\alpha \beta} & =\sum_{p=\text { odd }}^{N} \frac{p^{4}}{\left(p^{2}-4 \alpha^{2}\right)\left(p^{2}-4 \beta^{2}\right)} \\
& =\sum_{p=\text { odd }}^{N} 1+\sum_{p=\text { odd }}^{N} \frac{4 \beta^{2}}{p^{2}-4 \beta^{2}}+\sum_{p=\text { odd }}^{N} \frac{4 \alpha^{2}}{p^{2}-4 \alpha^{2}}+\sum_{p=\text { odd }}^{N} \frac{16 \alpha^{2} \beta^{2}}{\left(p^{2}-4 \beta^{2}\right)\left(p^{2}-4 \alpha^{2}\right)}
\end{aligned}
$$

For $N>>1$, the first term gives $\sum_{p=\text { odd }}^{N} 1=N / 2$, and the second and third terms vanish (cf. eq C4). For $\alpha \neq \beta$, the fourth term is rewritten as $\left\{4 \alpha^{2} \beta^{2} /\left(\beta^{2}-\alpha^{2}\right)\right\}\left\{\sum_{p=\text { odd }}^{N}\left(p^{2}-4 \beta^{2}\right)^{-1}-\sum_{p=\text { odd }}^{N}\left(p^{2}-4 \alpha^{2}\right)^{-1}\right\}$. This summation vanishes because of eq $\mathrm{C} 4$. On the other hand, for $\alpha=\beta$, the fourth term is calculated to be $\alpha^{2} \pi^{2} / 4$ with the aid of a mathematical formula, ${ }^{6}$

$$
\sum_{p=\text { odd }}^{\infty} \frac{1}{\left(p^{2}-x^{2}\right)^{2}}=-\frac{\pi}{8 x^{3}} \tan \left(\frac{\pi x}{2}\right)+\frac{\pi^{2}}{16 x^{2}} \sec ^{2}\left(\frac{\pi x}{2}\right)
$$

(For $x=2 \alpha$, this summation is equal to $\pi^{2} / 64 \alpha^{2}$.) Thus, eq C7 is finally rewritten as

$$
\dot{\mathbf{B}}_{\alpha}(t)=-\frac{4 \alpha^{2}}{\tau_{1}} \mathbf{B}_{\alpha}(t)-\frac{8 N}{\pi^{2} \tau_{1}} \sum_{\beta=0}^{\infty} \mathbf{B}_{\beta}(t)
$$

This equation is shown as eq $50 \mathrm{~b}$ (combined with eq 51 ) in the main text. 


\section{Appendix D. Solution of Eqs 52-54 in the Main Text.}

D1. Calculation of Laplace transformation. For a function of $t, f(t)$, we define the Laplace transformation as

$$
\tilde{f}(s) \equiv \int_{0}^{\infty} \mathrm{d} t f(t) \exp (-s t)
$$

Then, the time evolution equations, eqs 52-54 in the main text, can be rewritten for the Laplace transformation of $\mathbf{B}_{\alpha}(t)$ and $\mathbf{A}_{\alpha}(t)$ as

$$
\begin{array}{ll}
s \tilde{\mathbf{B}}_{0}(s)=\mathbf{B}_{0}(0)-\frac{1}{2 \tau_{1}} \tilde{\mathbf{Q}}(s) & \\
\left(s+\frac{4 \alpha^{2}}{\tau_{1}}+\frac{1}{\tau_{\mathrm{as}}}\right) \tilde{\mathbf{B}}_{\alpha}(s)-\frac{1}{\tau_{\mathrm{as}}} \tilde{\mathbf{A}}_{\alpha}(s)=\mathbf{B}_{\alpha}(0)-\frac{1}{\tau_{1}} \tilde{\mathbf{Q}}(s) & (1 \leq \alpha \leq N / 2) \\
\left(s+\frac{4 \alpha^{2}}{\tau_{1}}\right) \tilde{\mathbf{B}}_{\alpha}(s)=\mathbf{B}_{\alpha}(0)-\frac{1}{\tau_{1}} \tilde{\mathbf{Q}}(s) & (\alpha>N / 2) \\
\left(s+\frac{4 \alpha^{2}}{\tau_{1}}+\frac{1}{\tau_{\mathrm{ds}}}\right) \tilde{\mathbf{A}}_{\alpha}(s)-\frac{1}{\tau_{\mathrm{ds}}} \tilde{\mathbf{B}}_{\alpha}(s)=\mathbf{A}_{\alpha}(0) & (1 \leq \alpha \leq N / 2)
\end{array}
$$

with

$$
\tilde{\mathbf{Q}}(s)=\frac{8 N}{\pi^{2}} \sum_{\beta=0}^{\infty} \tilde{\mathbf{B}}_{\beta}(s)
$$

In the continuous treatment in the current analysis, the summation $\sum_{\beta=0}^{\infty} \tilde{\mathbf{B}}_{\beta}(s)$ is equal to zero (cf. Appendix B). However, the front factor in eq D5, $8 N / \pi^{2}$ with $N>>1$ ( or $N \rightarrow \infty$ ), allows $\tilde{\mathbf{Q}}(s)$ to remain non-zero. In this sense, $\tilde{\mathbf{Q}}(s)$ serves as the Lagrange factor. (If we erroneously set $\tilde{\mathbf{Q}}(s)=\mathbf{0}$, eq D2 does not reproduce the Rouse fluctuation of the normalized end-to-end vector of the linear chain, $\mathbf{B}_{0}(t)=N^{-1} \int_{0}^{N} \mathbf{u}^{[\mathrm{L}]}(n, t) \mathrm{d} n$.)

The factors $\mathbf{B}_{0}(0), \mathbf{B}_{\alpha}(0)$, and $\mathbf{A}_{\alpha}(0)$ appearing in eqs D2-D4 are the initial values of $\mathbf{B}_{0}(t)$, $\mathbf{B}_{\alpha}(t)$, and $\mathbf{A}_{\alpha}(t)$. For the affine deformation with respect to the shear strain $\gamma$ imposed at time 0 , $\mathbf{A}_{\alpha}(0)$ satisfies the simple initial condition specified for the shear components of the dyadic, as explained in Appendices B1 and B2 (eq D6a is identical to eq B6):

$$
\begin{aligned}
& \left\langle\mathbf{A}_{\alpha}(0) \mathbf{A}_{\beta}(0)\right\rangle_{x y}=\frac{2 b^{2} \gamma}{3 N} \delta_{\alpha \beta} \quad(\alpha, \beta \geq 1) \\
& \left\langle\mathbf{A}_{\alpha}(0) \mathbf{B}_{\beta}(0)\right\rangle_{x y}=0 \quad(\text { for any } \alpha, \beta)
\end{aligned}
$$


In contrast, no such simple initial condition is valid for $\mathbf{B}_{0}(0)$ and $\mathbf{B}_{\alpha}(0)$ because of the constraint, $\tilde{\mathbf{B}}_{0}(s)=-\sum_{\alpha=1}^{\infty} \tilde{\mathbf{B}}_{\alpha}(s)$; see Appendix B3. Thus, in the final stage of calculation, the initial amplitude $\mathbf{B}_{\alpha}(0)$ is rewritten in terms of the well-behaving $\boldsymbol{\Xi}_{p}(0)$, as explained in Appendix B3.

From eqs D3 and D4, we find

$$
\begin{aligned}
\tilde{\mathbf{B}}_{\alpha}(s)= & \left.\frac{1}{K+1}\left\{\frac{1}{\mathrm{~s}+\lambda_{\alpha}^{+}}+\frac{K}{\mathrm{~s}+\lambda_{\alpha}^{-}}\right\} \mathbf{B}_{\alpha}(0)-\frac{1}{\tau_{1}} \tilde{\mathbf{Q}}(s)\right\} & \\
& +\frac{K}{K+1}\left\{\frac{1}{\mathrm{~s}+\lambda_{\alpha}^{+}}-\frac{1}{\mathrm{~s}+\lambda_{\alpha}^{-}}\right\} \mathbf{A}_{\alpha}(0) & (1 \leq \alpha \leq N / 2) \\
\tilde{\mathbf{B}}_{\alpha}(s)= & \frac{1}{\mathrm{~s}+\lambda_{\alpha}^{+}}\left\{\mathbf{B}_{\alpha}(0)-\frac{1}{\tau_{1}} \tilde{\mathbf{Q}}(s)\right\} & (\alpha>N / 2) \\
\tilde{\mathbf{A}}_{\alpha}(s)= & \frac{1}{K+1}\left\{\frac{K}{\mathrm{~s}+\lambda_{\alpha}^{+}}+\frac{1}{\mathrm{~s}+\lambda_{\alpha}^{-}}\right\} \mathbf{A}_{\alpha}(0) & \\
& \left.+\frac{1}{K+1}\left\{\frac{1}{\mathrm{~s}+\lambda_{\alpha}^{+}}-\frac{1}{\mathrm{~s}+\lambda_{\alpha}^{-}}\right\} \mathbf{B}_{\alpha}(0)-\frac{1}{\tau_{1}} \tilde{\mathbf{Q}}(s)\right\} & (1 \leq \alpha \leq N / 2)
\end{aligned}
$$

where

$$
\lambda_{\alpha}^{+}=\frac{4 \alpha^{2}}{\tau_{1}}, \quad \lambda_{\alpha}^{-}=\frac{4 \alpha^{2}+r_{\mathrm{a}}+r_{\mathrm{d}}}{\tau_{1}}=\frac{4 \alpha^{2}+(K+1) r_{\mathrm{d}}}{\tau_{1}}
$$

and

$$
r_{\mathrm{a}}=\frac{\tau_{1}}{\tau_{\mathrm{as}}}, r_{\mathrm{d}}=\frac{\tau_{1}}{\tau_{\mathrm{ds}}}, K=\frac{[\mathrm{R}]_{\mathrm{eq}}}{[\mathrm{L}]_{\mathrm{eq}}}=\frac{r_{\mathrm{a}}}{r_{\mathrm{d}}}
$$

The factors $\lambda_{\alpha}^{+}, \lambda_{\alpha}^{-}, r_{\mathrm{a}}$, and $r_{\mathrm{d}}$ are identical to those defined by eqs 41 and 42 in the main text.

Now, we can utilize eq D7 to calculate the summation $\sum_{\alpha=1}^{\infty} \tilde{\mathbf{B}}_{\alpha}(s)$ as

$$
\begin{aligned}
\sum_{\alpha=1}^{\infty} \tilde{\mathbf{B}}_{\alpha}(s) & =\frac{1}{K+1} \sum_{\alpha=1}^{N / 2}\left(\frac{1}{\mathrm{~s}+\lambda_{\alpha}^{+}}+\frac{K}{\mathrm{~s}+\lambda_{\alpha}^{-}}\right) \mathbf{B}_{\alpha}(0)+\sum_{\alpha>N / 2}^{\infty} \frac{1}{\mathrm{~s}+\lambda_{\alpha}^{+}} \mathbf{B}_{\alpha}(0) \\
& +\frac{K}{K+1} \sum_{\alpha=1}^{N / 2}\left(\frac{1}{\mathrm{~s}+\lambda_{\alpha}^{+}}-\frac{1}{\mathrm{~s}+\lambda_{\alpha}^{-}}\right) \mathbf{A}_{\alpha}(0)-\tilde{H}(s) \tilde{\mathbf{Q}}(s)
\end{aligned}
$$

where $\tilde{H}(s)$ is a function of $s$ given by 


$$
\begin{aligned}
\tilde{H}(s) & =\frac{1}{\tau_{1}}\left\{\frac{1}{K+1} \sum_{\alpha=1}^{N / 2} \frac{1}{\mathrm{~s}+\lambda_{\alpha}^{+}}+\frac{K}{K+1} \sum_{\alpha=1}^{N / 2} \frac{1}{\mathrm{~s}+\lambda_{\alpha}^{-}}+\sum_{\alpha>N / 2}^{\infty} \frac{1}{\mathrm{~s}+\lambda_{\alpha}^{+}}\right\} \\
& \cong \frac{1}{\tau_{1}}\left\{\frac{1}{K+1} \sum_{\alpha=1}^{\infty} \frac{1}{\mathrm{~s}+\lambda_{\alpha}^{+}}+\frac{K}{K+1} \sum_{\alpha=1}^{\infty} \frac{1}{\mathrm{~s}+\lambda_{\alpha}^{-}}\right\} \\
& =\frac{1}{8(K+1)}\left\{-\frac{1}{\theta^{2}}+\frac{\pi \operatorname{coth}(\pi \theta)}{\theta}\right\}+\frac{K}{8(K+1)}\left\{-\frac{1}{\varphi^{2}}+\frac{\pi \operatorname{coth}(\pi \varphi)}{\varphi}\right\}
\end{aligned}
$$

with

$$
\theta=\frac{\sqrt{\tau_{1} s}}{2}, \quad \varphi=\frac{\sqrt{\tau_{1} s+(\mathrm{K}+1) r_{\mathrm{d}}}}{2}
$$

In derivation of eq D12 (third line), we have replaced $N / 2(>>1)$ in the summation ranges by $\infty$ (second line) and considered the factor $\alpha^{2}$ included in $\lambda_{\alpha}^{+}$and $\lambda_{\alpha}^{-}$(cf. eq D9) to utilize the following mathematical formula, ${ }^{6}$

$$
\sum_{\alpha \geq 1}^{\infty} \frac{1}{\alpha^{2}+x^{2}}=-\frac{1}{2 x^{2}}+\frac{\pi}{2 x} \operatorname{coth}(\pi x)
$$

From eqs D2, D5, and D11 and the relationship $\sum_{\alpha=0}^{\infty} \tilde{\mathbf{B}}_{\alpha}(s)=\mathbf{0}$, we can find an equation that determines $\tilde{\mathbf{Q}}(s)$ :

$$
\begin{aligned}
\mathbf{0}= & \frac{1}{s} \mathbf{B}_{0}(0)-\frac{1}{2 \tau_{1} s} \tilde{\mathbf{Q}}(s) \\
& +\frac{1}{K+1} \sum_{\beta=1}^{N / 2}\left(\frac{1}{\mathrm{~s}+\lambda_{\beta}^{+}}+\frac{K}{\mathrm{~s}+\lambda_{\beta}^{-}}\right) \mathbf{B}_{\beta}(0)+\sum_{\beta>N / 2}^{\infty} \frac{1}{\mathrm{~s}+\lambda_{\beta}^{+}} \mathbf{B}_{\beta}(0) \\
& +\frac{K}{K+1} \sum_{\beta=1}^{N / 2}\left(\frac{1}{\mathrm{~s}+\lambda_{\beta}^{+}}-\frac{1}{\mathrm{~s}+\lambda_{\beta}^{-}}\right) \mathbf{A}_{\beta}(0)-\tilde{H}(s) \tilde{\mathbf{Q}}(s)
\end{aligned}
$$

From eq D15 combined with eqs D9, D12, D13 and the relationship $\tilde{\mathbf{B}}_{0}(s)=-\sum_{\alpha=1}^{\infty} \tilde{\mathbf{B}}_{\alpha}(s)$, we can express $\tilde{\mathbf{Q}}(s)$ in terms of the initial amplitudes, $\mathbf{B}_{\alpha}(0)$ and $\mathbf{A}_{\alpha}(0)$ with $\alpha \geq 1$ :

$$
\begin{aligned}
\tilde{\mathbf{Q}}(s)= & -2 \tau_{1} \tilde{J}(s) \sum_{\beta=1}^{N / 2}\left(\frac{1}{K+1} \frac{\beta^{2}}{\beta^{2}+\theta^{2}}+\frac{K}{K+1} \frac{\beta^{2}+(\mathrm{K}+1) r_{\mathrm{d}} / 4}{\beta^{2}+\varphi^{2}}\right) \mathbf{B}_{\beta}(0) \\
& -2 \tau_{1} \tilde{J}(s) \sum_{\beta>N / 2}^{\infty} \frac{\beta^{2}}{\beta^{2}+\theta^{2}} \mathbf{B}_{\beta}(0) \\
& -2 \tau_{1} \tilde{J}(s) \frac{K}{K+1} \sum_{\beta=1}^{N / 2}\left(\frac{\beta^{2}}{\beta^{2}+\theta^{2}}-\frac{\beta^{2}+(K+1) r_{\mathrm{d}} / 4}{\beta^{2}+\varphi^{2}}\right) \mathbf{A}_{\beta}(0)
\end{aligned}
$$

with 


$$
\begin{aligned}
\tilde{J}(s) & =\frac{1}{2 \tau_{1} s \tilde{H}(s)+1} \\
& =\left\{\frac{1}{K+1} \pi \theta \operatorname{coth}(\pi \theta)+\frac{K}{K+1} \frac{\pi \theta^{2} \operatorname{coth}(\pi \varphi)}{\varphi}-\frac{K}{K+1}\left(\frac{\theta^{2}}{\varphi^{2}}-1\right)\right\}^{-1}
\end{aligned}
$$

Here, $\theta$ and $\varphi$ are functions of $s$, as specified by eq D13.

The orientational correlation function, the basic function of our interest, can be expressed in terms of $\mathbf{B}_{\alpha}(t)$ and $\mathbf{A}_{\alpha}(t)$ with $\alpha \geq 1$ (without explicitly involving $\mathbf{B}_{0}(t)=-\sum_{\alpha=1}^{\infty} \mathbf{B}_{\alpha}(t)$; cf. eq 49 in the main text). Thus, we hereafter focus on $\mathbf{B}_{\alpha}(t)$ and $\mathbf{A}_{\alpha}(t)$ with $\alpha \geq 1$. As noted from eqs D7 and D8, calculation of $\mathbf{B}_{\alpha}(t)$ and $\mathbf{A}_{\alpha}(t)$ requires us to analyze the poles of the kernel function $\tilde{J}(s)$ included in $\tilde{\mathbf{Q}}(s)$ (cf. eqs D16 and D17). In the followings, we firstly make this pole analysis and then calculate $\mathbf{B}_{\alpha}(t)$ and $\mathbf{A}_{\alpha}(t)$.

\section{D2. Pole Analysis for $\tilde{J}(s)$}

As can be noted from eq D17, $\tilde{J}(s)$ has first order poles at $s=s_{k}=-\mu_{k}^{2} / \tau_{1}\left(\right.$ i.e., $\left.1 / \tilde{J}\left(s_{k}\right)=0\right)$, with $\mu_{k}$ being a normalized, real and positive eigenvalue that satisfies

$$
-\frac{\pi \cot \left(\mu_{k} \pi / 2\right)}{\mu_{k}}+\frac{K \pi \operatorname{coth}\left((\pi / 2) \sqrt{(K+1) r_{d}-\mu_{k}^{2}}\right)}{\sqrt{(K+1) r_{d}-\mu_{k}^{2}}}-2 K\left\{\frac{1}{(K+1) r_{d}-\mu_{k}^{2}}+\frac{1}{\mu_{k}^{2}}\right\}=0
$$

(Note in eq D17 that $[\pi \theta \operatorname{coth}(\pi \theta)]_{\theta \rightarrow 0}=1$ so that $\tilde{J}(s)$ has no pole at $s=0$.) For $\mu_{k}^{2}<(K+1) r_{d}$, both nominator and denominator in the second term are real numbers, and eq D18 is valid as it is. On the other hand, for $\mu_{k}^{2}>(K+1) r_{d}$, the $\operatorname{coth}\left\{(\pi / 2) \sqrt{(K+1) r_{d}-\mu_{k}^{2}}\right\}$ and $\sqrt{(K+1) r_{d}-\mu_{k}^{2}}$ factors appearing therein are rewritten as $-i \cot \left\{(\pi / 2) \sqrt{\mu_{k}^{2}-(K+1) r_{d}}\right\}$ and $i \sqrt{\mu_{k}^{2}-(K+1) r_{d}}$, respectively.

The first order pole at $s=s_{k}$ is associated with a residue $R_{k}$. This $R_{k}$ is calculated, with the aid of l'Hôpital's rule, as

$$
R_{k}=\left[\left(s-s_{k}\right) \tilde{J}(s)\right]_{s \rightarrow s_{k}}=\frac{1}{\left[\frac{\mathrm{d}}{\mathrm{d} s}\left(\frac{1}{\tilde{J}(s)}\right)\right]_{s \rightarrow s_{k}}}
$$

Thus, we find, from eqs D18 and D19:

$$
R_{k}=\frac{8}{\tau_{1} \pi^{2}} \frac{K+1}{R_{k 1}+R_{k 2}}
$$

with 


$$
\begin{aligned}
& R_{k 1}=\frac{1}{\sin ^{2}\left(\mu_{k} \pi / 2\right)}+\frac{K \mu_{k}^{2}}{\left((\mathrm{~K}+1) r_{\mathrm{d}}-\mu_{k}^{2}\right) \sinh ^{2}\left((\pi / 2) \sqrt{(\mathrm{K}+1) r_{\mathrm{d}}-\mu_{k}^{2}}\right)} \\
& R_{k 2}=\left\{1+\frac{\mu_{k}^{2}}{(\mathrm{~K}+1) r_{\mathrm{d}}-\mu_{k}^{2}} \int \frac{\cot \left(\mu_{k} \pi / 2\right)}{\left(\mu_{k} \pi / 2\right)}+\frac{4 K}{\mu_{k}^{2} \pi^{2}}\left(2-\frac{\mu_{k}^{2}}{(\mathrm{~K}+1) r_{\mathrm{d}}-\mu_{k}^{2}}\right)\right\}
\end{aligned}
$$

(For $\mu_{k}^{2}>(K+1) r_{d}$, the $\sinh ^{2}\left((\pi / 2) \sqrt{(\mathrm{K}+1) r_{\mathrm{d}}-\mu_{k}^{2}}\right)$ factor appearing in eq D21a is rewritten as $\left.-\sin ^{2}\left((\pi / 2) \sqrt{\mu_{k}^{2}-(\mathrm{K}+1) r_{\mathrm{d}}}\right) \cdot\right)$

Utilizing the eigenvalue $\mu_{k}$ and the residue $R_{k}$ explained above, we can express the kernel function $J(t)$ in the time domain as

$$
J(t)=\sum_{k \geq 1} R_{k} \exp \left(-\Lambda_{k} t\right) \text { with } \Lambda_{k}=\frac{\mu_{k}^{2}}{\tau_{1}}
$$

D3. Calculation of $\mathbf{B}_{\alpha}(t)$ and $\mathbf{A}_{\alpha}(t)$.

Making Laplace inversion of eq D16, we obtain

$$
\begin{aligned}
\mathbf{Q}(t)= & -\frac{2 \tau_{1}}{K+1} \sum_{\beta \geq 1}^{N / 2} \mathbf{B}_{\beta}(0) \int_{0}^{t} \mathrm{~d} t^{\prime}\left\{\lambda_{\beta}^{+} \exp \left(-\lambda_{\beta}^{+} t^{\prime}\right)+K \lambda_{\beta}^{-} \exp \left(-\lambda_{\beta}^{-} t^{\prime}\right)\right\} J\left(t-t^{\prime}\right) \\
& -2 \tau_{1} \sum_{\beta>N / 2}^{\infty} \mathbf{B}_{\beta}(0) \int_{0}^{t} \mathrm{~d} t^{\prime} \lambda_{\beta}^{+} \exp \left(-\lambda_{\beta}^{+} t^{\prime}\right) J\left(t-t^{\prime}\right) \\
& -\frac{2 K \tau_{1}}{K+1} \sum_{\beta \geq 1}^{N / 2} \mathbf{A}_{\beta}(0) \int_{0}^{t} \mathrm{~d} t^{\prime}\left\{\lambda_{\beta}^{+} \exp \left(-\lambda_{\beta}^{+} t^{\prime}\right)-\lambda_{\beta}^{-} \exp \left(-\lambda_{\beta}^{-} t^{\prime}\right)\right\} J\left(t-t^{\prime}\right)
\end{aligned}
$$

With the aid of eq D22, we can calculate the integrals in eq D23 to find

$$
\mathbf{Q}(t)=\sum_{\beta \geq 1}^{\infty} \mathbf{B}_{\beta}(0) b_{\beta}^{[\mathrm{Q}]}(t)+\sum_{\beta \geq 1}^{N / 2} \mathbf{A}_{\beta}(0) a_{\beta}^{[\mathrm{Q}]}(t)
$$

with

$$
\begin{array}{ll}
b_{\beta}^{[\mathrm{Q}]}(t)=-\frac{2 \tau_{1}}{K+1} \sum_{k=1}^{\infty} R_{k}\left\{\lambda_{\beta}^{+} \omega\left(t ; \Lambda_{k}, \lambda_{\beta}^{+}\right)+K \lambda_{\beta}^{-} \omega\left(t ; \Lambda_{k}, \lambda_{\beta}^{-}\right)\right\} & (1 \leq \beta \leq N / 2) \\
b_{\beta}^{[\mathrm{Q}]}(t)=-2 \tau_{1} \sum_{k=1}^{\infty} R_{k} \lambda_{\beta}^{+} \omega\left(t ; \Lambda_{k}, \lambda_{\beta}^{+}\right) & (N / 2<\beta<\infty) \\
a_{\beta}^{[\mathrm{Q}]}(t)=-\frac{2 K \tau_{1}}{K+1} \sum_{k=1}^{\infty} R_{k}\left\{\lambda_{\beta}^{+} \omega\left(t ; \Lambda_{k}, \lambda_{\beta}^{+}\right)-\lambda_{\beta}^{-} \omega\left(t ; \Lambda_{k}, \lambda_{\beta}^{-}\right)\right\} & (1 \leq \beta \leq N / 2)
\end{array}
$$

where the function $\omega\left(t ; \lambda, \lambda^{\prime}\right)$ is defined as

$$
\omega\left(t ; \lambda, \lambda^{\prime}\right)=\frac{\exp \left(-\lambda^{\prime} t\right)-\exp (-\lambda t)}{\lambda-\lambda^{\prime}}
$$


with

$$
\omega(t ; \lambda, \lambda) \equiv\left[\frac{\exp \left(-\lambda^{\prime} t\right)-\exp (-\lambda t)}{\lambda-\lambda^{\prime}}\right]_{\lambda^{\prime} \rightarrow \lambda}=t \exp (-\lambda t)
$$

Making Laplace inversion of eq D7 and D8 with the aid of eqs D24 and D25, we finally find expressions of $\mathbf{B}_{\alpha}(t)$ (for the linear chain) and $\mathbf{A}_{\alpha}(t)$ (for the ring chain):

$$
\begin{aligned}
& \mathbf{B}_{\alpha}(t)=\sum_{\beta=1}^{N / 2} \mathbf{B}_{\beta}(0) b_{\alpha \beta}^{[\mathrm{B}]}(t)+\sum_{\beta>N / 2}^{\infty} \mathbf{B}_{\beta}(0) b_{\alpha \beta}^{*[\mathrm{~B}]}(t)+\sum_{\beta=1}^{N / 2} \mathbf{A}_{\beta}(0) a_{\alpha \beta}^{[\mathrm{B}]}(t) \quad(1 \leq \alpha<\infty) \\
& \mathbf{A}_{\alpha}(t)=\sum_{\beta=1}^{N / 2} \mathbf{B}_{\beta}(0) b_{\alpha \beta}^{[\mathrm{A}]}(t)+\sum_{\beta>N / 2}^{\infty} \mathbf{B}_{\beta}(0) b_{\alpha \beta}^{*[\mathrm{~A}]}(t)+\sum_{\beta=1}^{N / 2} \mathbf{A}_{\beta}(0) a_{\alpha \beta}^{[\mathrm{A}]}(t) \quad(1 \leq \alpha \leq N / 2)
\end{aligned}
$$

The functional form of $b_{\alpha \beta}^{[\mathrm{cc}]}(t), b_{\alpha \beta}^{*[\mathrm{c}]}(t)$, and $a_{\alpha \beta}^{[\mathrm{c}]}(t) \quad$ (with $\mathrm{c}=\mathrm{A}$ and $\mathrm{B}$ ) is explicitly shown in eqs 56 , 57 , and 59 in the main text.

In relation to this functional form, we should note that eqs D7 and D8 include product terms, $(s+\lambda)^{-1} \tilde{\mathbf{Q}}(s)$ with $\lambda=\lambda_{\alpha}^{+}$and/or $\lambda_{\alpha}^{-}$. Laplace inversion of these terms gives a convolution, $\int_{0}^{t} \mathrm{~d} t^{\prime} \exp \left\{-\lambda\left(t-t^{\prime}\right)\right\} \mathbf{Q}\left(t^{\prime}\right)$, and this convolution is included in $b_{\alpha \beta}^{[\mathrm{cc}}(t), b_{\alpha \beta}^{*[\mathrm{cc}]}(t)$, and $a_{\alpha \beta}^{[\mathrm{cc}]}(t)$. Because $\mathbf{Q}(t)$ is given as a sum of the functions $\omega\left(t ; \lambda, \lambda^{\prime}\right)$ (cf. eqs D24-D26), $b_{\alpha \beta}^{[\mathrm{c}]}(t), b_{\alpha \beta}^{*[\mathrm{c}]}(t)$, and $a_{\alpha \beta}^{[\mathrm{c}]}(t)$ (shown in eqs 56, 57, and 59 in the main text) are expressed in terms of the convolution function $\Omega\left(t ; \lambda^{*}, \lambda, \lambda^{\prime}\right)$ defined by

$$
\begin{aligned}
& \Omega\left(t ; \lambda^{*}, \lambda, \lambda^{\prime}\right) \\
& \equiv \int_{0}^{t} \mathrm{~d} t^{\prime} \exp \left(-\lambda^{*}\left(t-t^{\prime}\right)\right) \omega\left(t^{\prime} ; \lambda, \lambda^{\prime}\right) \\
& =\frac{1}{\lambda-\lambda^{\prime}}\left\{\frac{\exp \left(-\lambda^{\prime} t\right)-\exp \left(-\lambda^{*} t\right)}{\lambda^{*}-\lambda^{\prime}}-\frac{\exp (-\lambda t)-\exp \left(-\lambda^{*} t\right)}{\lambda^{*}-\lambda}\right\}
\end{aligned}
$$

For some special cases of $\lambda^{*}, \lambda$, and $\lambda^{\prime}, \Omega\left(t ; \lambda^{*}, \lambda, \lambda^{\prime}\right)$ is simplified as

$$
\begin{aligned}
\Omega\left(t ; \lambda^{*}, \lambda, \lambda\right)= & {\left[\Omega\left(t ; \lambda^{*}, \lambda, \lambda^{\prime}\right)\right]_{\lambda^{\prime} \rightarrow \lambda} } \\
& =\frac{t \exp (-\lambda t)}{\lambda^{*}-\lambda}-\frac{\exp (-\lambda t)-\exp \left(-\lambda^{*} t\right)}{\left(\lambda^{*}-\lambda\right)^{2}} \\
\Omega\left(t ; \lambda^{*}, \lambda, \lambda^{*}\right) & =\left[\Omega\left(t ; \lambda^{*}, \lambda, \lambda^{\prime}\right)\right]_{\lambda^{\prime} \rightarrow \lambda^{*}} \\
& =\frac{t \exp \left(-\lambda^{*} t\right)}{\lambda-\lambda^{*}}+\frac{\exp (-\lambda t)-\exp \left(-\lambda^{*} t\right)}{\left(\lambda^{*}-\lambda\right)^{2}}
\end{aligned}
$$

and

$$
\Omega(t ; \lambda, \lambda, \lambda)=\left[\Omega\left(t ; \lambda^{*}, \lambda, \lambda^{\prime}\right)\right]_{\lambda^{\prime}, \lambda^{*} \rightarrow \lambda}=\frac{1}{2} t^{2} \exp (-\lambda t)
$$


The function $\Omega\left(t ; \lambda^{*}, \lambda, \lambda^{\prime}\right)$ is zero at $t=0$ for any value of $\lambda^{*}, \lambda$, and $\lambda^{\prime}$. This feature of $\Omega\left(t ; \lambda^{*}, \lambda, \lambda^{\prime}\right)$ becomes very important when we examine the initial amplitudes of the cosine modes $\mathbf{B}_{\alpha}(0)$ and $\mathbf{A}_{\alpha}(0)$, as explained in section 3-1 in the main text.

\section{D4. Additional comment.}

All results of the above analysis were obtained from eqs 52-54 in the main text, with eq $53 \mathrm{~b}$ including no decay term, $-\mathbf{B}_{\alpha}(t) / \tau_{\mathrm{as}}$. The analysis can be made similarly when this term is added to eq $53 \mathrm{~b}$, although this addition leads to an unreasonable situation as explained in section 2-4-3. The results for this case remain the same as shown in eqs 55-59, except that $\lambda_{\alpha}^{*}$ appearing therein is replaced by $\left(4 \alpha^{2}+K r_{\mathrm{d}}\right) / \tau_{1}$. 


\section{Appendix E. Behavior of Linear and Ring Chains in Slow Reaction Limit.}

In the slow reaction limit $\left(r_{\mathrm{d}}=\tau_{1} / \tau_{\mathrm{ds}} \rightarrow 0\right)$, the kernel function (eq D17), the eigenvalue equation (eq D18), and the expression of the residue (eqs D20 and D21) are simplified as

$$
\begin{aligned}
& \tilde{J}(s)=\frac{1}{\pi \theta \operatorname{coth}(\pi \theta)} \text { with } \theta=\frac{\sqrt{\tau_{1} s}}{2} \\
& \cot \left(\mu_{k} \pi / 2\right)=0, \mu_{k}=k \text { (odd integer) } \\
& R_{k}=\frac{8}{\tau_{1} \pi^{2}} \text { (independent of } k \text { ) }
\end{aligned}
$$

In this limit, the relaxation rates shown in eqs 41 and 60 in the main text converge as

$$
\begin{aligned}
& \lambda_{\alpha}^{+}, \lambda_{\alpha}^{-}, \lambda_{\alpha}^{*} \rightarrow \lambda_{\alpha} \equiv \frac{4 \alpha^{2}}{\tau_{1}}(\alpha=\text { integer }) \\
& \Lambda_{k} \rightarrow \frac{k^{2}}{\tau_{1}}(k=\text { odd integer })
\end{aligned}
$$

For this case, eq 59 in the main text gives $b_{\alpha \beta}^{[\mathrm{A}]}(t)=0, b_{\alpha \beta}^{*}{ }^{* \mathrm{~A}]}(t)=0$, and $a_{\alpha \beta}^{[\mathrm{A}]}(t)=\delta_{\alpha \beta} \exp \left(-4 \alpha^{2} t / \tau_{1}\right)$, which correctly reproduces the pure Rouse behavior of $\mathbf{u}^{[\mathrm{R}]}(n, t)$ of the ring chain in the absence of reaction (eqs 22 and 23 in the main text).

For the linear chain in the slow reaction limit, eqs 56 and 57 in the main text give

$$
\begin{array}{ll}
b_{\alpha \beta}^{[\mathrm{B}]}(t)=\delta_{\alpha \beta} \exp \left(-\lambda_{\alpha} t\right)+\frac{16 \lambda_{\beta}}{\tau_{1} \pi^{2}} \sum_{k=\text { odd }}^{\infty} \Omega\left(t ; \lambda_{\alpha}, \Lambda_{k}, \lambda_{\beta}\right) & (1 \leq \alpha \leq N / 2) \\
b_{\alpha \beta}^{*[\mathrm{~B}]}(t)=\frac{16 \lambda_{\beta}}{\tau_{1} \pi^{2}} \sum_{k=\text { odd }}^{\infty} \Omega\left(t ; \lambda_{\alpha}, \Lambda_{k}, \lambda_{\beta}\right) & (1 \leq \alpha \leq N / 2) \\
a_{\alpha \beta}^{[\mathrm{B}]}(t)=0 & (1 \leq \alpha \leq N / 2)
\end{array}
$$

and

$$
\begin{array}{ll}
b_{\alpha \beta}^{[\mathrm{B}]}(t)=\frac{16 \lambda_{\beta}}{\tau_{1} \pi^{2}} \sum_{k=\text { odd }}^{\infty} \Omega\left(t ; \lambda_{\alpha}, \Lambda_{k}, \lambda_{\beta}\right) & (N / 2<\alpha<\infty) \\
b_{\alpha \beta}^{*[\mathrm{~B}]}(t)=\delta_{\alpha \beta} \exp \left(-\lambda_{\alpha} t\right)+\frac{16 \lambda_{\beta}}{\tau_{1} \pi^{2}} \sum_{k=\text { odd }}^{\infty} \Omega\left(t ; \lambda_{\alpha}, \Lambda_{k}, \lambda_{\beta}\right) & (N / 2<\alpha<\infty) \\
a_{\alpha \beta}^{[\mathrm{B}]}(t)=0 & (N / 2<\alpha<\infty)
\end{array}
$$

Substituting eqs E5 and E6 in eqs 67 and 68 in the main text and considering the expression of $\Omega$ (eq D29), we find 


$$
\mathbf{u}_{\text {odd }}^{[\mathrm{L}]}(n, t)=\sum_{p=\text { odd }}^{N} \boldsymbol{\Xi}_{p}^{*}(t) \sin \left(\frac{p \pi n}{N}\right)
$$

with

$$
\boldsymbol{\Xi}_{p}^{*}(t)=\sum_{q=\mathrm{odd}}^{N} \boldsymbol{\Xi}_{q}(0) \xi_{p q}(t)
$$

and

$$
\xi_{p q}(t)=I_{p q}(t)+I I_{p q}(t)+I I I_{p q}(t)
$$

The functions $I_{p q}(t), I I_{p q}(t)$, and $I I I_{p q}(t)$ included in eq E8 are defined as

$$
\begin{aligned}
& I_{p q}(t)=\frac{64 q}{p \pi^{2}} \sum_{\alpha=1}^{\infty} \frac{\alpha^{2}}{\left(p^{2}-4 \alpha^{2}\right)\left(q^{2}-4 \alpha^{2}\right)} \exp \left(-\lambda_{\alpha} t\right) \\
& I_{p q}(t)=\frac{256 q}{p \pi^{4}} \sum_{\alpha=1}^{\infty} \sum_{\beta=1}^{\infty} \frac{16 \alpha^{2} \beta^{2}}{\left(p^{2}-4 \alpha^{2}\right)\left(q^{2}-4 \beta^{2}\right)} \frac{\exp \left(-\lambda_{\beta} t\right)-\exp \left(-\lambda_{\alpha} t\right)}{4 \alpha^{2}-4 \beta^{2}} \times\left\{\sum_{k=\mathrm{odd}}^{\infty} \frac{1}{k^{2}-4 \beta^{2}}\right\} \\
& I I I_{p q}(t)=\frac{256 q}{p \pi^{4}} \sum_{\alpha=1}^{\infty} \sum_{k=\mathrm{odd}}^{\infty} \frac{4 \alpha^{2}}{\left(p^{2}-4 \alpha^{2}\right)} \frac{\exp \left(-\Lambda_{k} t\right)-\exp \left(-\lambda_{\alpha} t\right)}{k^{2}-4 \alpha^{2} \times} \times\left\{\sum_{\beta=1}^{\infty} \frac{4 \beta^{2}}{\left(q^{2}-4 \beta^{2}\right)\left(k^{2}-4 \beta^{2}\right)}\right\}
\end{aligned}
$$

We can make the summation appearing in Eq 9 in a stepwise way with the aid of the following mathematical formulae. ${ }^{6}$ (Equation E10a is equivalent to eq C4 in Appendix C, and eqs E10b and E10c are identical to eqs 79 and 80 in the main text.)

$$
\begin{aligned}
& \sum_{k=\text { odd }}^{\infty} \frac{1}{k^{2}-x^{2}}=\frac{\pi}{4 x} \tan \left(\frac{\pi x}{2}\right) \\
& \sum_{\alpha=1}^{\infty} \frac{1}{\alpha^{2}-x^{2}}=\frac{1}{2 x^{2}}-\frac{\pi}{2 x} \cot (\pi x) \\
& \sum_{\alpha=1}^{\infty} \frac{1}{\left(\alpha^{2}-x^{2}\right)^{2}}=\frac{\pi^{2}}{4 x^{2}} \operatorname{cosec}^{2}(\pi x)+\frac{\pi}{4 x^{3}} \cot (\pi x)-\frac{1}{2 x^{4}}
\end{aligned}
$$

Comparing eqs E9b and E10a, we find

$$
I I_{p q}(t)=0
$$

From eqs E10b and E10c, we can rewrite eq E9c as 


$$
\begin{aligned}
I I I_{p q}(t) & =\frac{256 q}{p \pi^{4}} \sum_{\alpha=1}^{\infty} \sum_{k=\text { odd }}^{\infty} \frac{4 \alpha^{2}}{\left(p^{2}-4 \alpha^{2}\right)} \frac{\exp \left(-\Lambda_{k} t\right)-\exp \left(-\lambda_{\alpha} t\right)}{k^{2}-4 \alpha^{2}} \times\left\{\frac{\pi^{2}}{16} \delta_{q k}\right\} \\
& =\frac{64 q}{p \pi^{2}} \sum_{\alpha=1}^{\infty} \frac{\alpha^{2}}{\left(p^{2}-4 \alpha^{2}\right)\left(q^{2}-4 \alpha^{2}\right)}\left\{\exp \left(-\Lambda_{q} t\right)-\exp \left(-\lambda_{\alpha} t\right)\right\}
\end{aligned}
$$

Finally, substituting eqs E11 and E12 into eq E8 and considering eq E9a, we obtain

$$
\begin{aligned}
\xi_{p q}(t) & =\frac{64 q}{p \pi^{2}}\left\{\sum_{\alpha=1}^{\infty} \frac{\alpha^{2}}{\left(p^{2}-4 \alpha^{2}\right)\left(q^{2}-4 \alpha^{2}\right)}\right\} \exp \left(-\Lambda_{q} t\right) \\
& =\delta_{p q} \exp \left(-\Lambda_{p} t\right) \\
& =\delta_{p q} \exp \left(-p^{2} t / \tau_{1}\right)
\end{aligned}
$$

(The summation in eq E13 is conducted with the aid of eqs E10b and E10c.)

Equations E13 and E7 indicates that the pure Rouse contribution of the odd sine modes to the subchain bond vector of the linear chain, $\mathbf{u}_{\text {odd }}^{[\mathrm{L}]}(n, t)=\sum_{p=\text { odd }}^{N} \boldsymbol{\Xi}_{p}(0) \exp \left(-p^{2} t / \boldsymbol{\tau}_{1}\right) \sin (p \pi n / N)$, is recovered in the slow reaction limit. Furthermore, the pure Rouse contribution of the even sine mode is also recovered in the slow reaction limit, as noted from eq 39 (in the main text) combined with eq E4a. Thus, our formulation correctly reproduces the pure Rouse behavior of the linear chain in that limit.

It should be noted that the pure Rouse behavior of both ring and linear chains is recovered for $r_{\mathrm{d}}=\tau_{1} / \tau_{\mathrm{ds}} \rightarrow 0$ (and $\lambda_{\alpha}^{+}, \lambda_{\alpha}^{-}, \lambda_{\alpha}^{*} \rightarrow 4 \alpha^{2} / \tau_{1}$; eq E4a) irrespective of the equilibrium constant, $K=$ $[\mathrm{R}]_{\mathrm{eq}} /[\mathrm{L}]_{\mathrm{eq}}=r_{\mathrm{a}} / r_{\mathrm{d}}$. The linear and ring population does not change with time, but conversion of a particular ring chain into a linear chain requires a time $\tau_{\mathrm{ds}}$ on average, and conversion of a particular linear chain into a ring chain, a time $\tau_{\mathrm{as}}=\tau_{\mathrm{ds}} / K$. The chemical equilibration, reflected in $K(=$ $\exp (-\Delta G / R T)$ with $\Delta G$ being the free energy difference between the linear and ring chains), is achieved through these conversions and requires a time $\tau_{\mathrm{eq}}=\max \left\{\tau_{\mathrm{as}}, \tau_{\mathrm{ds}}\right\}$, whereas the Rouse relaxation completes within a much shorter time $\tau_{1}\left(<<\tau_{\text {eq }}\right)$. For this reason, the equilibrium constant does not affect the recovery of the pure Rouse behavior for the case of $r_{\mathrm{d}} \rightarrow 0$.

\section{References}

1) Watanabe H.; Inoue, T. "Conformational dynamics of Rouse chains during creep/recovery processes: a review", J. Phys. Condensed Matter 2005, 17, R607-R636.

2) Watanabe, H.; Inoue, T.; Matsumiya, Y., "Transient Conformational Change of Bead-Spring Ring Chain During Creep Process", Macromolecules 2006, 39, 5419-5426. 
3) Watanabe, H.; Matsumiya, Y.; Masubuchi, Y.; Urakawa, O.; Inoue, Y., "Viscoelastic Relaxation of Rouse Chains undergoing Head-to-Head Association and Dissociation: Motional Coupling through Chemical Equilibrium", Macromolecules 2015, 48, 3014-3030.

4) Doi, M.; Edwards, S. F. The Theory of Polymer Dynamics, Oxford: Clarendon, 1986.

5) Watanabe, H., "Viscoelasticity and dynamics of entangle polymers", Prog. Polym. Sci. 1999, 24, 1253-1403.

6) Moriguchi, S.; Udagawa, K.; Hitotsumatsu, S. Mathematical Formula, Iwanami: Tokyo, 1986. 